\title{
Design of an Acquisition System for Monitoring the Current of a GEM Detector
}

\author{
Rabí Soto-Camachoํㅡㄹ David Pinto Avendaño ${ }^{1}$, Sergio Vergara-Limon ${ }^{2}$, \\ Maria-Aurora Vargas-Treviño ${ }^{2}$, Guy Paic ${ }^{3}$ \\ ${ }^{1}$ Benemérita Universidad Autónoma de Puebla, \\ Facultad de Ciencias de la Computación, Puebla, México \\ ${ }^{2}$ Benemérita Universidad Autónoma de Puebla, \\ Facultad de Ciencias de la Electrónica, Puebla, México \\ ${ }^{3}$ Universidad Nacional Autónoma de México, \\ Instituto de Ciencias Nucleares, CDMX, México
} rabi87_soto22@hotmail.com, dpinto@cs.buap.mx, svergara2@hotmail.com,
auroravargast@hotmail.com, Guy.Paic@cern.ch

\begin{abstract}
In the development of this work we describe the design proposal of a useful tool for the monitoring of the GEM detector panels in order to measure the current consumption and in this way to know the useful life of the detector, due to in theory the GEM doesn't produce discharges but in real life there are some small discharges (nano and micro amperes) that deteriorate the detector. In this proposal it is also contemplated to design a software to analyze the interaction of electromagnetic fields within a waveguide that represents the pathways within a printed circuit board using the study of electromagnetic theory in the development of a simulator, which will allow to analyze the effects of these fields in the propagation of the signals that are obtained and to determine if the data obtained are adequate to allow their subsequent analysis.
\end{abstract}

Keywords. Electromagnetic interferences, current monitor, EMI analysis.

\section{Introduction}

A GEM (gas electron multiplier) is made of a plate (50 microns) of Kapton sheet, a copper coating on each side, surface perforated, each acting as a channel for electron multiplication. Each hole has a bi-conical structure with an outer diameter of 50 microns and a step of 140 microns (see Fig. 1). 

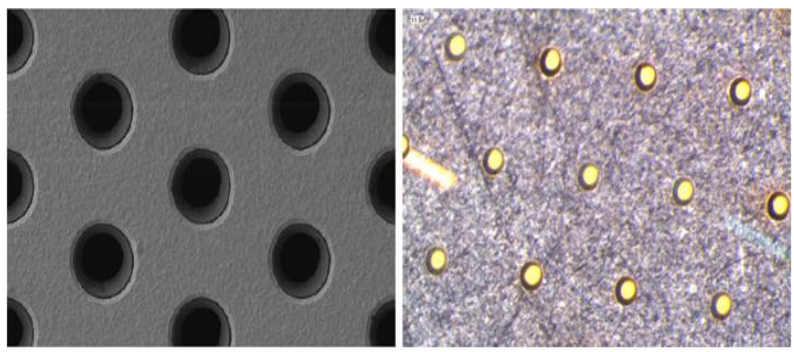

Fig. 1. Microscopic Image of a GEM.

A charged particle or a photon that interacts in the gas of the drift region produces an ionization cluster of positive ions and electrons. Due to the applied electric field, the group of positive ions of the drift goes towards the cathode, while the group of electrons goes towards the holes of the first sheet of GEM.

Here the charge multiplies and while the amplified charge towards the second GEM is frustrated a large percentage of the positive ions produced in the holes is captured by the upper GEM electrode. Thus, the multiplication channel is released quickly and groups of electrons multiply. The gain is proportional to the sum of the GEM voltages (see Fig. 2) [1].

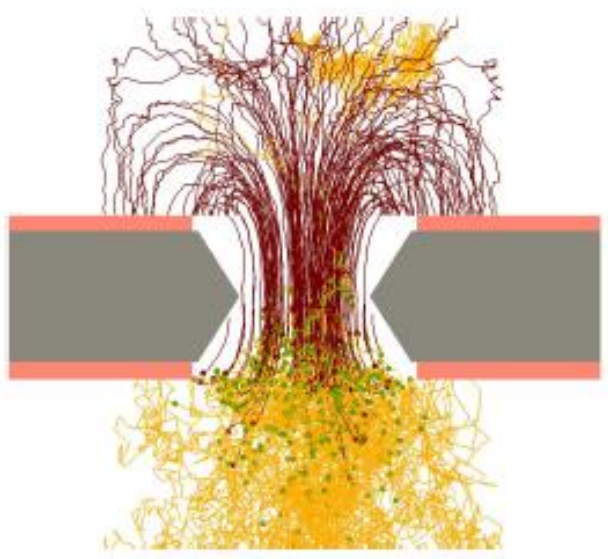

Fig. 2. GEM Operation.

To carry out the measurement of the current consumption of these detectors requires specialized devices for this. This document proposes the development of a current monitor to monitor this consumption, as well as the development of a software to carry out the analysis of electromagnetic fields that can be presented on the monitor. 


\subsection{Current Monitor}

The current monitor is an electronic circuit that serves to monitor current consumption for the system, nowadays there are some devices in the market, but they are not suitable for monitoring each channel of the GEMs and to record the current variations of the detector due to the features of the detector. A prototype was found in the litherature but our proposal in the design is different so the features of the prototype could be useful to compare the performance design [2]. We need to develop a prototype that must be capable to measure the small discharges (nano and micro amperes) and also not interfere in the normal work of the detector. The proposed system is shown in figure 3 .

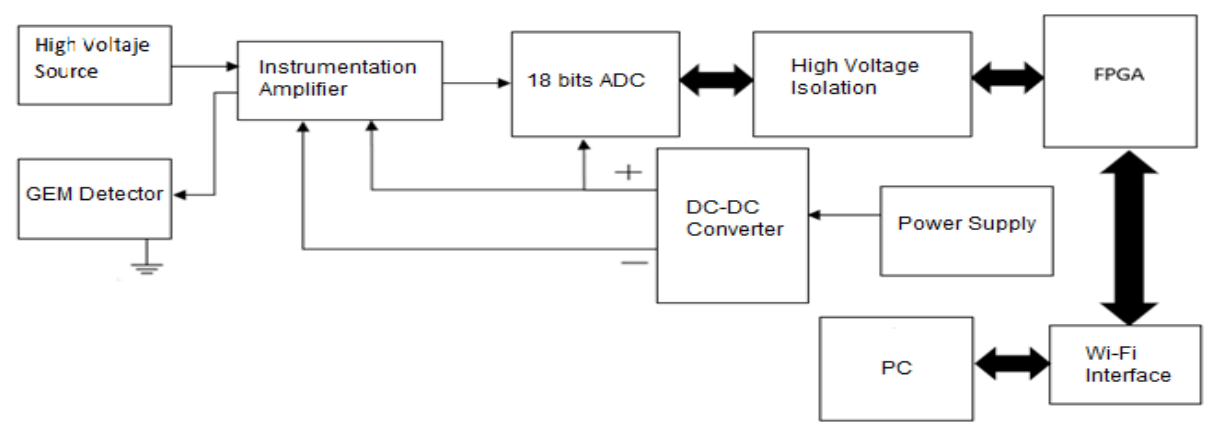

Fig. 3. Current Monitor Block Diagram.

The system must be connected to the high voltage and the GEM detector for which high voltage connectors are required.

The voltage levels necessary to use the current meter card are obtained from the use of a DC-DC converter, in this case in addition to conditioning the card voltage, it allows isolating the high and low voltage supplies. Later a voltage regulator is used in order to condition the operating voltage of the ADC.

Currently there are some software for the analysis of electromagnetic fields, but their costs are very high for this reason a key part of the design of this tool is to provide a tool for analysis at a lower cost. On the other hand, when carrying out the experiments an enormous quantity of data is generated, which must be processed for its study, for this reason the development of this research topic is centered as the first point in the development of a software for analysis of electromagnetic fields to be able to study the effects of said fields on the acquired signals and thus be able to obtain adequate data for their analysis. As a second point of this research project once the analysis is performed and appropriate data are generated, these must be processed to obtain those essential for study and storage $[3,4,15,16]$.

\section{Proposal for Developing}

For the development of this topic, a block diagram with the work methodology to be followed is presented below: 


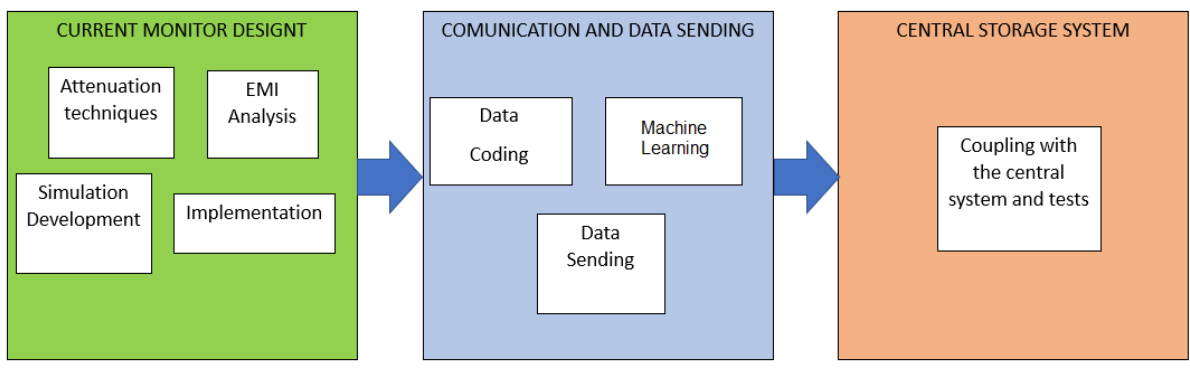

Fig. 4. Proposal Block Diagram.

In the previous diagram, 3 action blocks are presented that define the process to be followed in this work.

A. In the design stage of the Current Monitor various activities are carried out in order to obtain a functional system as a result.

- The study of the techniques for the reduction of electromagnetic interference is done in order to have the adequate knowledge of how electromagnetic fields interact within a medium and thus be able to attenuate them in order to obtain desired signals without noise.

- The analysis of electromagnetic interferences is done to see how the signals are transmitted in different media, as well as to know how they are attenuated in these.

- The software design for the analysis of electromagnetic fields is done in order to determine how these affect the signals and to perform an analysis of it to obtain adequate values of signals for further analysis through the use of data cleansing techniques.

- The instrumentation of the current monitor is performed in order to visualize its operation and observe the interferences that may occur due to the electromagnetic fields inside the circuit and outside.

B. In the Data Processing stage, the following stages are presented:

- In the data coding stage, the $8 \mathrm{~b} / 10 \mathrm{~b}$ coding is performed in order to have a standard coding pattern with the data.

- he Machine Learning stage is carried out because when making measurements with the system, an important quantity of data is required, therefore it is necessary to use machine learning techniques to classify and find patterns in these.

- The stage of sending data is necessary since it is necessary to store the data once classified so that it can be reused for another type of subsequent analysis.

C. The storage stage is performed in order to have the available data to be able to perform some kind of processing or subsequent analysis.

For the design stage of the current monitor, the study of techniques and theories has been carried out in order to obtain a functional device. 


\subsection{Propagation of Electromagnetic Waves}

The electromagnetic radiation and its propagation are described by the Maxwell's equations with the electric field perpendicular to the magnetic field. These equations determine the effects of transmission, reflection, attenuation and dispersion of the propagated wave, the Maxwell equations are shown in Table 1

Table 1. Maxwell's equations.

\begin{tabular}{|c|c|c|}
\hline Differential form & Integral form & \\
\hline \hline$\nabla \cdot \vec{D}=\rho_{v}$ & $\oint D \cdot d s=\int_{v} \rho_{v} d v$ & Gauss Law \\
\hline \hline$\nabla \cdot \vec{B}=0$ & $\oint_{w} B \cdot d s=0$ & $\begin{array}{c}\text { Gauss Law } \\
\text { (H) }\end{array}$ \\
\hline \hline$\nabla x \vec{E}=-\frac{\partial \vec{B}}{\partial t}$ & $\int_{L} E \cdot d l=-\frac{\partial}{\partial t} \int_{s} B \cdot d s$ & $\begin{array}{c}\text { Faraday's } \\
\text { Law }\end{array}$ \\
\hline \hline$\nabla x \vec{H}=\vec{J}+\frac{\partial \vec{D}}{\partial t}$ & $\int_{L} H \cdot d l=\int_{s}\left(J+\frac{\partial D}{\partial t}\right) \cdot d s$ & $\begin{array}{c}\text { Ampere's } \\
\text { Law }\end{array}$ \\
\hline
\end{tabular}

where we have:

$\vec{E}=$ Vector electric intensity or electric field $[\mathrm{V} / \mathrm{m}]$.

$\vec{B}=$ Magnetic flow or magnetic induction $\left[T=W b / m^{2}\right]$.

$\vec{H}$ = Vector of magnetic intensity or magnetic field $[\mathrm{A} / \mathrm{m}]$.

$\vec{I}=$ Current intensity or conduction $\left[\mathrm{A} / \mathrm{m}^{2}\right]$.

$\vec{D}=$ Electric flow or displacement current $\left[\mathrm{C} / \mathrm{m}^{2}\right]$.

$\mathrm{q}=$ Electric charge.

The description of this implies that Faraday's law indicates that a magnetic field can produce a current in a closed path if the magnetic flux is changing in time. In relation to Lenz's law, the voltage induced by the magnetic flux has such a polarity that the current established in a closed path gives rise to a flux that opposes the magnetic flux change. Ampere's law states that the magnetic field is generated by both the conduction current and the displacement currents. The first equation of Gauss's law states that the electric flow that passes through any closed surface is equal to the total charge enclosed in the surface. Gauss's law for the magnetic field states that the magnetic flux lines are closed and do not end in a magnetic charge.

The propagation of electromagnetic waves depends on two factors:

- Of the nature of the medium (permittivity or dielectric constant, magnetic permeability and conductivity).

- Of the electromagnetic wave frequency. 
The system of equations that relate and describe the electromagnetic propagation with the nature of the medium are:

$$
\begin{aligned}
& \vec{D}=\varepsilon \vec{E} \\
& \vec{B}=\mu \vec{H} \\
& \vec{J}=\sigma \vec{E} \\
& \varepsilon=\varepsilon_{0} \varepsilon_{r} \\
& \mu=\mu_{0} \mu_{r}
\end{aligned}
$$

where:

$\varepsilon=$ Dielectric permittivity $[\mathrm{F} / \mathrm{m}]$.

$\mu=$ Magnetic permittivity $[\mathrm{H} / \mathrm{m}]$.

$\varepsilon_{0}, \mu_{0}=$ They correspond to the values of the vacuum.

$\varepsilon_{r}, \mu_{r}=$ They correspond to the values relative to the medium.

$\sigma=$ Electric conductivity $[\mathrm{S} / \mathrm{m}=1 / \Omega \mathrm{m}]$.

The dielectric permittivity is a measure of the degree of resistance of the medium to a load flow and is determined by the relationship between the electric displacement and the intensity of the field that produces it.

The Magnetic permeability is the ability of a medium to make way for magnetic field lines and is determined by the relationship between magnetic induction and the external magnetic field $[5,6,8,11-14,17]$.

\subsection{Electromagnetic Interference in Electronic Circuits}

Electromagnetic interference (EMI) can be defined as electromagnetic signals that disturb (unintentionally) the normal operation of an electrical or electronic system.

The types of interference, measurement methods and tolerated limits are specified by international standards and are established according to the frequency band of interference.

- Low frequency disturbances: ( $\mathrm{f}<10 \mathrm{KHz}$ ), emitted by the network and power supplies.

- Disturbances in band of 10-150 KHz: By impulses of intensity and transitory phenomena of tension switches, etc.

- Disturbances in band of $150 \mathrm{KHz}$ to $30 \mathrm{MHz}$ : By impulses of intensity and transitory phenomena of tension switches, the propagation is by radiation and coupling.

- Disturbances in band of $30 \mathrm{MHz}$ to $300 \mathrm{MHz}$ : Propagation by radiation.

- Disturbances in band of $500 \mathrm{MHz}$ to $18 \mathrm{GHz}$ : It propagates by radiation and usually appears in communications equipment or switching logic circuits.

By an EMI it can be understood as the presence of unwanted voltage or current that can appear in a device as a result of the operation of another device or natural phenomenon. 
The coupling between systems consists of the interaction and disturbance of one device depending on another.

The interference can be radiated or conducted, when the noise originates in a source or travels through the air is called EMI radiation, when the noise driven travels through a conductor, as a power line. The noise may have been radiated, deposited in the lines and then driven $[6,7,9,10-13]$.

\section{Conclusions and Future Work}

By continuously monitoring the current consumption, the degradation of the GEM can be known since the GEM in initial conditions has a specific current consumption and the more current consumption is visualized, it gives us an idea of how degraded the material is.

It is possible to design and implement a device that allows monitoring the current consumption of the order of nanoamperes using electromagnetic interference attenuation techniques in order to determine if what we are monitoring is noise or a defined signal.

By knowing the techniques and theories about the attenuation of electromagnetic interference it is possible to develop a useful software tool that allows us to know the degree of attenuation of the interferences using certain techniques.

The detection of phenomena of electromagnetic type will allow to improve the state of the art in the design of printed circuits since having a tool that allows us to know the attenuation of electromagnetic interferences can avoid the remaking of circuits due to faults in the medium.

\section{References}

1. Santos-Rodrigues, A.T.S.: Gas Electron Multiplier. CERN, Sitio web: https://kt.cern/technologies/gas-electron-multiplier (2016)

2. Schweda K.: Alice TPC GEM High-Voltage System: Engineering Design Review. Sitio Web: https://indico.cern.ch/event/677082/contributions/2772028/attachments/1562708/ 2464403/hv-edr-schweda.pdf (2017)

3. Software FEKO para análisis de circuitos, disponible en: http://www.indielec.es/fekocms-4-50-376/ (2017)

4. Software ZUKEN para análisis de circuitos, disponible en: https://www.zuken.com/en (2017)

5. Reitz, J., Milford, F., Christy, R.: Fundamentos de la teoría electromagnética. Tercera edición, Editorial Addison Wesley

6. Resnick, R.: Physics Vol. 2, Tercera edición, Editorial, Continental S.A. de C.V. Mexico.

7. Félix, M.A., Salazar, D., Valenzuela, J.A., Márquez, H.: Análisis de propagación de campos electromagnéticos en guías de onda por medio un método para barrido de parámetro V. SOMI Congreso de Instrumentación, XVIII, disponible en: $\mathrm{ftp} / / /$ www.ece.buap.mx/pub/Secretaria_Academica/ACADEMIA/ASISTENCIA $\% 20 \mathrm{~A} \%$ 20EVENTOS\%20ACAD\%C9MICOS\%20Y\%20PERMISOS\%20TEMPORALES/CON GRESO\%20SOMI\%20XVIII/PDF/Microondas/71.pdf, 1-11 (2005) 
8. Chattopadhyay, S.: Generation of magnetic and electric field measurement and its analysis. Proceedings of the International Confeerence on Electromagrietic Interference and Compatibility, De INCEMIC-99, Sitio Web: https://ieeexplore.ieee.org/document/ 871631, 219-224 (1999)

9. Wang, C., Chen, Y., Zhang, G., Zhou, Z.: Design of Printed-Circuit Board Rogowski Coil for Highly Accurate Current Measurement. In International Conference on Mechatronics and Automation, Sitio web: http://ieeexplore.ieee.org/document/4304180/ (2007)

10.Sato, T., Matsunaga, T., Maeda, H., Watanabe, M.: Application Examples of Electromagnetic Field Analysis at Fujitsu. FUJITSU Sci. Tech Sitio web: https://www.fujitsu.com/global/documents/about/resources/publications/fstj/archives/ vol 48(4) (2012)

11.Park, H.H., Jang, H.T., Park, H.B.: Simulation and Design of a PCB-Chassis System for Reducing Radiated Emissions. ACES Journal, 26, 679-687 (2011)

12.De Burgh, G.: Making EMI Analysis Part of the Design Process. COTS Journal Sitio web: http://archive.cotsjournalonline.com/articles/view/100226 (2004)

13.Gao, S., Zhang, Z., Bo, R.: Research on the magnetic property of high-speed PCB design. Journal of Chemical and Pharmaceutical Research Sitio web: http://www.jocpr.com/articles/research-on-the-magnetic-property-of-highspeed-pcbdesign.pdf (2015)

14.Zumbahlen, H.: Printed Circuit-Board Design Issues. Linear Circuit Design Handbook. sitio web: https://www.oreilly.com/library/view/linear-circuit-design/9780750687034/, 1201-1297 (2008)

15.Antillanca, E.H., Cerda, N.G.: Propuesta Metodológica para el Desarrollo de Software de Investigación. Universidad de Santiago de Chile Sitio web: http://sitios.diinf.usach.cl/kddp/files/2012/10/Paper-Propuesta-de-M\%C3\%A9todo-paraSw-de-Investigaci\%C3\%B3n-Infonor-2010.pdf (2012)

16.Chavarriaga, L.J., Arboleda, J.H.: Modelo de Investigación en Ingeniería del Software: Una propuesta de investigación tecnológica. Universidad San Buenaventura Sitio web: http://www.emn.fr/x-info/harbol07/MIFISIS2004.pdf (2004)

17.Mologni, J.: Compatibilidad electromagnética. Sitio web: http://www.esss.com.br/blog/ es/2016/08/compatibilidade-electromagnetica/ (2016) 